\title{
Understanding of Corrosion Phenomena: Process, Mechanism, and Method
}

\author{
Rokuro Nishimura, ${ }^{1}$ Tooru Tsuru, ${ }^{2}$ Toshiaki Ohtsuka, ${ }^{3}$ Nobuyoshi Hara, ${ }^{4}$ \\ En-Hou Han, ${ }^{5}$ and Osama Alyousif ${ }^{6}$ \\ ${ }^{1}$ Graduate School of Engineering, Osaka Prefecture University, Sakai, Osaka 599-8531, Japan \\ ${ }^{2}$ Graduate School of Science and Engineering, Tokyo Institute of Technology, O-okayama, Meguro-ku, Tokyo 152-8552, Japan \\ ${ }^{3}$ Faculty of Engineering, Hokkaido University, Hokkaido, Sapporo 060-8628, Japan \\ ${ }^{4}$ Graduate School of Engineering, Tohoku University, Aoba-ku, Miyagi, Sendai 980-8579, Japan \\ ${ }^{5}$ Institute of Metal Research, Chinese Academy of Sciences, 62 Wencui Road, Liaoning, Shenyang 110016, China \\ ${ }^{6}$ Mechanical Engineering Department, College of Engineering \& Petroleum, Kuwait University, P.O. Box 5969, Safat 13060, Kuwait
}

Correspondence should be addressed to Rokuro Nishimura, nishimu@mtr.osakafu-u.ac.jp

Received 8 July 2012; Accepted 8 July 2012

Copyright ( $) 2012$ Rokuro Nishimura et al. This is an open access article distributed under the Creative Commons Attribution License, which permits unrestricted use, distribution, and reproduction in any medium, provided the original work is properly cited.

Corrosion phenomena of materials are very complicated in order that corrosion reactions and/or processes depend largely upon the material/environment systems. The damage of the materials caused by the corrosion phenomena has led to the loss in energy and resources, the instability of human life, the decline in the reliability of the infrastructure, and so on. To overcome them, recent advances in the understanding of corrosion phenomena and the mechanisms have been introduced for new aspects and perspective to study the roles of anion species and films formed in corrosion behavior, the factors influencing SCC (stress corrosion cracking) susceptibility, hydrogen permeation behavior associated with hydrogen embrittlement, and so on. These have led to the development of a higher corrosion resistant material, a more suitable inhibitor and corrosion prevention method, and to the reasonable judgment of the material selection in a given corrosive environment. Therefore, this special issue was focused on recent developments in stress corrosion cracking, advances in corrosion resistant materials, role of atomic hydrogen in hydrogen embrittlement, the stability and strength of the films formed, new aspects of localized corrosion (pitting corrosion and crevice corrosion), recent developments in inhibitors, new methods for elucidating corrosion behavior, and so on.

Several papers described the corrosion protection, atomic modeling of corrosion phenomena, the corrosion products exposed with the long-range term at the atmospheric environment and the corrosion behavior of advance material (Ni-based intermetallic compound). T. Ohtsuka reviewed the corrosion protection of steels by the coating of the conducting polymers which are polyaniline, polypyrrole, and polythiophene. He described that they work as a strong oxidant, inducing the potential shift to the noble direction. C. D. Taylor reviewed and considered the atomistic modeling of corrosion events such as the metal-water interface, the reaction of water, competitive adsorption, the structure of a metallic surface, and so on. F. Q-Li et al. investigated many bronze wares of Qin Dynasty excavated from tombs at Xinfeng town using SEM/EDS, XRD and RM and obtained valuable information on the conservation of the bronze wares. S. O. Adeosun et al. investigated the corrosion behavior of as-cast and retrogressed-reaged Al-Mg alloys in solutions of $\mathrm{NaCl}, \mathrm{FeCl}_{3}$, and so on. They pointed out that $\mathrm{Mg}_{2} \mathrm{Si}$ crystals being anodic relative to the matrix affected the corrosion behavior. The corrosion behavior of $\mathrm{Ni}_{3} \mathrm{Al} / \mathrm{Ni}_{3} \mathrm{~V}$ twophase intermetallic compounds was investigated in various acidic solutions by using the immersion test and SEM by G. Priyotomo et al. They found that the preferential dissolution of $\left(\mathrm{Ll}_{2}+\mathrm{D}_{22}\right)$ phase took place and the corrosion behavior depended largely upon anion species.

The other papers described the localized corrosion behavior such as the intergranular corrosion, pitting corrosion and SCC M. E. Arikan et al. determined the susceptibility to intergranular corrosion of UNS 31803-type duplex stainless 
steel, which was aged at various times with $725^{\circ} \mathrm{C}$, using electrochemical reactivation method and weight loss test. They found that the degree of sensitization was related to chromium- and molybdenum-depleted areas. N. Hara et al. studied the pitting corrosion resistance of $316 \mathrm{~L}$-type stainless steel. They concluded that the enhancement in pitting corrosion resistance was due to the beneficial effect of removing $\mathrm{MnS}$ inclusions from the steel surface rather than the modification effect of the chemical composition of passive films. The SCC of A516 pressure vessel steel was investigated by slow strain rate tests in $\mathrm{H}_{2} \mathrm{O}-\mathrm{CO}-\mathrm{CO}_{2}$ solutions by J. W. van der Merwe. It was found that $\mathrm{CO}$ component played an important role in initiation of SCC, that is, the inhibition of corrosion reaction. H. Nakano et al. investigated the SCC property of $\mathrm{Al}-\mathrm{Mg}$ alloy processed by equal-channel angular pressing (ECAP) by a slow strain technique in $3 \% \mathrm{NaCl}$ of $\mathrm{pH} 4$ at $303 \mathrm{~K}$. They found that the decrease in the SCC susceptibility was attributed to an improvement in corrosion resistance afforded by ECAP. O. M. Alyousif et al. investigated the effect of applied stress on environment induced cracking (EIC) of Al alloy (5052) in $0.5 \mathrm{M} \mathrm{NaCl}$ solution using a constant load method. They concluded that the steady state elongation rate became a parameter for predicting time to failure and the EIC was induced by SCC, not by HE (hydrogen embrittlement).

\section{Rokuro Nishimura Tooru Tsuru Toshiaki Ohtsuka \\ Nobuyoshi Hara En-Hou Han Osama Alyousif}



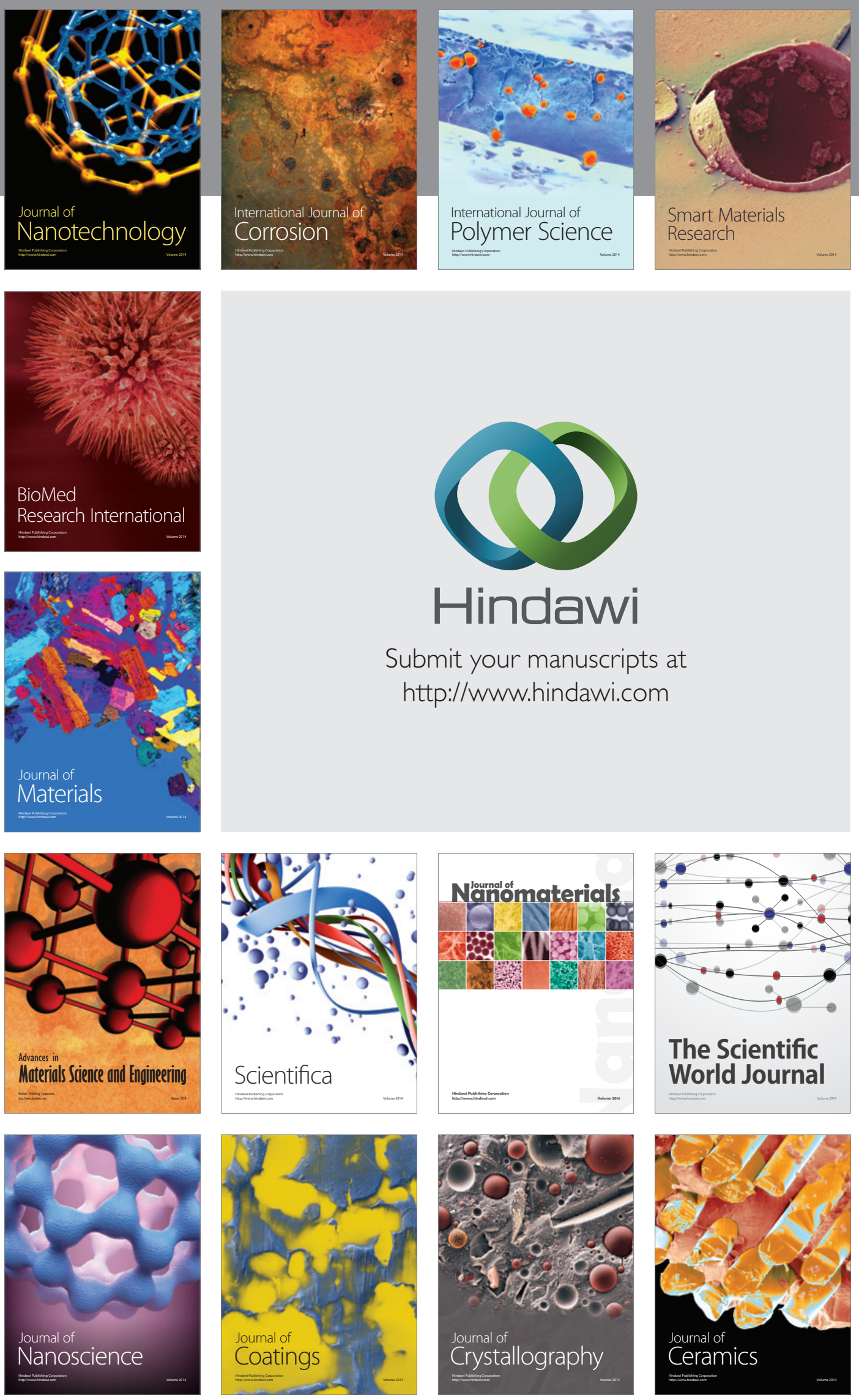

The Scientific World Journal

Submit your manuscripts at

http://www.hindawi.com

\section{World Journal}

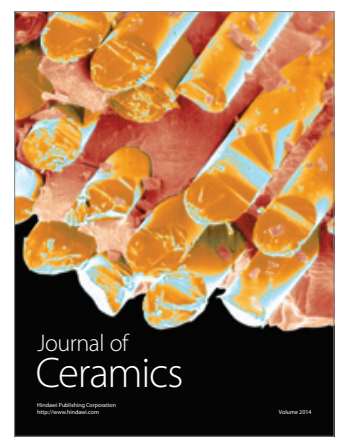

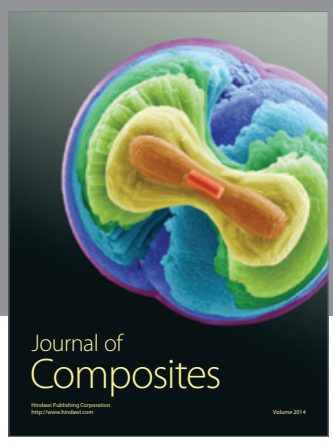
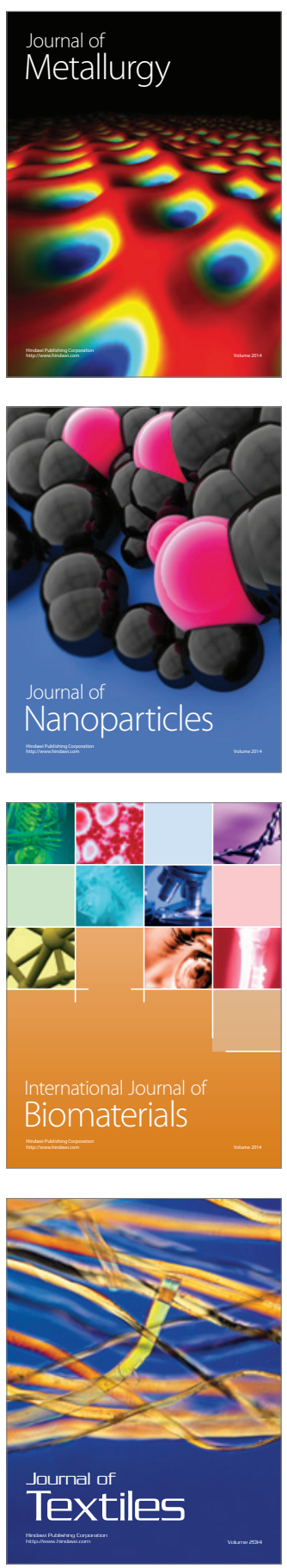